\title{
Optimal Quantum Estimation of Loss in Bosonic Channels
}

\author{
Alex Monras ${ }^{1}$ and Matteo G. A. Paris ${ }^{2, *}$ \\ ${ }^{1}$ Grup de Fisica Teòrica \& IFAE, Universitat Autònoma de Barcelona, Bellaterra E-08193, Spain \\ ${ }^{2}$ Dipartimento di Fisica dell'Università di Milano, Milano I-20133, Italia
}

(Received 7 February 2007; published 17 April 2007)

\begin{abstract}
We address the estimation of the loss parameter of a bosonic channel probed by Gaussian signals. We derive the ultimate quantum bound with precision and show that no improvement may be obtained by having access to the environmental degrees of freedom. We find that, for small losses, the variance of the optimal estimator is proportional to the loss parameter itself, a result that represents a qualitative improvement over the shot-noise limit. An observable based on the symmetric logarithmic derivative is obtained, which attains the ultimate bound and may be implemented using Gaussian operations and photon counting.
\end{abstract}

DOI: 10.1103/PhysRevLett.98.160401

PACS numbers: 03.65.Ta, 03.67.Hk, 42.50.Dv

In all the branches of physics, many quantities of interest are not directly accessible, either in principle, like the measurement of all fields [1], or due to experimental impediments. In these cases, one should resort to indirect measurements, inferring the value of the quantity of interest from its influence on a given probe. This is basically a parameter estimation problem whose solution, i.e., the determination of the most precise estimator, unavoidably involves an optimization procedure [2]. Indeed, the central result in classical estimation is the so-called Cramer-Rao inequality, which sets a lower bound on the variance of any estimator in terms of the Fisher information. When quantum systems are involved, the optimal measurement to detect an unobservable quantity may be found using tools from quantum estimation theory [3,4]. The quantum version of the Cramer-Rao inequality has been established [36] and the lower bound imposed by the quantum Fisher information has been shown to be achievable asymptotically [6]. In turn, this result permits us to write a parameterbased uncertainty relation for unobservable quantities [6,7].

Quantum estimation theory has been mostly applied to find optimal measurements and, in turn, to evaluate the corresponding lower bounds on precision, for the estimation of parameters imposed by unitary transformations. For bosonic systems these include single-mode phase [8-10], displacement [11], and squeezing [12,13] as well as twomode transformations, e.g., bilinear coupling [14]. Concerning open quantum systems and nonunitary processes, QET has been applied only to finite dimensional systems [15], to optimally estimate the noise parameter of depolarizing [16] or amplitude-damping [17] channels. On the other hand, to the best of our knowledge, QET has been not exploited to determine the optimal way of estimating parameters of a lossy bosonic channel. Needless to say, besides fundamental interest, quantum limits to the estimation of bosonic lossy channels are extremely relevant for applications, as, for example, absorption measurements and characterization of optical media [18-20].
The precision of a measurement corresponds to the smallest value of the parameter that can be discriminated. In several quantum mechanical schemes, precision is improved by the more efficient use of the resources employed in the measurement process [21,22]. A relevant example is the quadratic increase of the precision in phase estimation by squeezed states. On the other hand, despite the improvement in the resource-precision trade-off, known estimation schemes are characterized by a threshold value, under which the parameter cannot be measured. The results described in this Letter, besides being the first example of optimized parameter estimations for nonunitary maps, show a novel feature of quantum-limited measurements: the asymptotic variance scales with the parameter itself. Therefore, our results may be applied to arbitrarily small values of the parameter, with a fixed relative variance.

The scheme we are going to consider is the following: the dynamics of a bosonic quantum system is governed by a Lindblad master equation of the form

$$
\dot{\varrho}=\frac{\gamma}{2} \mathcal{L}[a] \varrho,
$$

which results from the interaction of the system with an external environment, as, for example, a bath of oscillators. We want to estimate the value of the loss parameter $\gamma$, and to this purpose a set of identically prepared signals (probe state) are sent through the channel and measured at the output. We denote by $\varrho_{0}$ the input state and by $\varrho_{\gamma}=$ $E_{\gamma}\left(\varrho_{0}\right)$ the state at the output of the channel, $E_{\gamma}$ being the map associated to the evolution (1). An estimation strategy for $\gamma$ consists of three ingredients: a suitable input signal, a measurement at the output, and an inference rule to extract the value of the parameter from the experimental sample $\boldsymbol{\chi}$, i.e., the set of measurement outcomes. The ultimate goal of choosing an estimation strategy is to find a scheme that maximizes precision with the constraint of a finite fixed amount of energy impinged into the channel. In particular, we address the following questions: (1) Which is the best probe state? (2) Which is the optimal measure- 
ment that should be performed at the output? (3) Which is the attainable precision? (4) Can the precision be improved by accessing the environment degrees of freedom, e.g., the bath of oscillators?

The quantum state at the output of the channel may be formally written as $\varrho_{\gamma}=E_{\gamma}\left(\varrho_{0}\right)=\exp (\gamma t / 2 \mathcal{L}[a]) \varrho_{0}$, whereas the constraint of finite probe energy reads as follows: $\operatorname{Tr}\left[\varrho_{0} a^{\dagger} a\right]=\bar{n},\left[a, a^{\dagger}\right]=1$ being the bosonic mode operators. We focus attention on Gaussian probe states and consider a zero-temperature environment without squeezing; i.e., we focus on lossy channels described by a superoperator of the form $\mathcal{L}[a] \varrho=2 a^{\dagger} \varrho a-$ $a^{\dagger} a \varrho-\varrho a^{\dagger} a$. Given the evolution time (length) $t$, the aim is to estimate as precisely as possible the parameter $\gamma$ in the Lindblad Master Eq. (1). In the following we consider the parametrization $\exp (-\gamma t)=\cos ^{2} \phi$ and seek for the best estimation strategy for $\phi$. We denote the probe state as $\varrho_{0}$ and the evolved state as $\varrho_{\phi}$ with the master equation that rewrites as $d \varrho / d \phi=\tan \phi \mathcal{L}[a] \varrho$. Our goal is to determine the optimal probe state $\varrho_{0}$ and the optimal measurement, i.e., the probability operator-value measure $O_{\chi}$ in order to form the estimator $\hat{\phi}$ to infer the value of $\phi$ from the set of outcomes $\boldsymbol{\chi}$. Notice that, with our estimation problem uniparametric, the optimal measurement can be realized using individual measurements on separate (subsequent) preparations [23], so that the expectation value of the estimator can be written as $\mathbb{E}_{\phi}[\hat{\phi}]=$ $\sum_{\chi} \hat{\phi}(\boldsymbol{\chi}) \operatorname{Tr}\left[\left(\varrho_{\phi}\right)^{\otimes N} O_{\chi}\right]$. We focus attention on asymptotically unbiased estimators, i.e., those for which $\mathbb{E}_{\phi}[\hat{\phi}] \rightarrow \phi$ as $N \rightarrow \infty$. Those are interesting because as the number of experimental data increases, all systematic errors go to zero. The variance of an unbiased estimator is bounded by the Cramer-Rao inequality [2], $\operatorname{Var}_{\phi}[\hat{\phi}] \geq \frac{1}{F(\phi)}$, where $F(\phi)$ is the Fisher information of the measurement. $F(\phi)$ is additive, i.e., the total Fisher information of measurements on multiple copies of $\varrho_{\phi}$ is the sum of the individual ones. It is worth mentioning that the so-called maximum likelihood estimator asymptotically attains this bound for large numbers of identical repeated samplings of a probability distribution. In such case the asymptotic variance scales as $1 / N$, i.e., $\operatorname{Var}_{\phi}[\hat{\phi}] \rightarrow[N F(\phi)]^{-1}$. The Fisher Information $F(\phi)$ is bounded from above by the quantum Fisher information (QFI) $F(\phi) \leq H(\phi)$ which, in turn, provides a measure of the ultimate precision available with a given quantum state. QFI is additive too, and thus we have $\operatorname{Var}_{\phi}[\hat{\phi}] \geq \frac{1}{N H(\phi)}$, where $H(\phi)$ can be expressed in terms of the symmetric logarithmic derivative, (SLD) $\Lambda(\phi)$. SLD is implicitly defined as the Hermitian operator that satisfies

$$
\frac{d \varrho_{\phi}}{d \phi}=\frac{1}{2}\left[\varrho_{\phi} \Lambda(\phi)+\Lambda(\phi) \varrho_{\phi}\right],
$$

from which the QFI can be computed as $H(\phi)=$ $\operatorname{Tr}\left[\varrho_{\phi} \Lambda(\phi)^{2}\right] . \Lambda(\phi)$ is an observable which depends on $\phi$ and, analogously to the classical LD, its expectation value is zero, i.e., $\operatorname{Tr}\left[\Lambda(\phi) \varrho_{\phi}\right]=0$. A biased $\Lambda(\hat{\phi})$ reveals to which extent the measured state differs from $\varrho_{\hat{\phi}}$. In addition, it has been proved [5] that an optimal measurement can be obtained by projecting onto one-dimensional eigenspaces of $\Lambda(\phi)$, i.e., a measurement for which the Fisher information is maximized, $F(\phi)=H(\phi)$. Upon this considerations, in the following we consider the onestep adaptive strategy [10,23], in which one makes a rough estimate $\hat{\phi}_{0}$ on a vanishing fraction of copies $N^{\delta}, 1 / 2<$ $\delta<1$, and then measures $\Lambda\left(\hat{\phi}_{0}\right)$ on the remaining copies in order to refine the estimate $\hat{\phi}$.

Notice that the Cramer-Rao inequality can be regarded as a Heisenberg relation for parameter estimation. In the case of pure states and unitary evolution of the form $U(\phi)=\exp (i G \phi)$ we have $H(\phi)=4\langle\Delta G\rangle^{2}$, where $\langle\Delta G\rangle^{2}$ is the uncertainty of the generator $G$ on the state used as a probe. Overall, we get the inequality $\operatorname{Var}_{\phi}[\hat{\phi}] \times$ $\langle\Delta G\rangle^{2} \geq 1 /(4 N)$, which represents a parameter-based uncertainty relation for unobservable quantities $[6,7]$.

Any Gaussian state of a single bosonic mode may be represented as a thermal state $\rho_{\mu}$ under the action of a squeezing and a displacement operation, i.e., $\varrho=D(\alpha) \times$ $S(\zeta) \rho_{\mu} S^{\dagger}(\zeta) D^{\dagger}(\alpha)$, where $\alpha=s e^{i \theta}$ is the displacement amplitude and $\zeta=r e^{-2 i \varphi}$ is the squeezing parameter; $D(\alpha)=\exp \left(\alpha a^{\dagger}-\alpha^{*} a\right), \quad S(\zeta)=\exp \left(\frac{1}{2} \zeta^{2} a^{\dagger 2}-\frac{1}{2} \zeta^{* 2} a^{2}\right)$. The thermal state can be expressed in terms of its purity as $\rho_{\mu}=2 \mu /(1+\mu)[(1-\mu) /(1+\mu)]^{a^{\dagger} a}$. At this point, some considerations about the choice of a probe state are in order. We aim at finding the Gaussian state $\varrho_{0}$ which, under the action of the amplitude-damping channel, is mapped onto $\varrho_{\phi}$ with highest QFI. Of course, if an arbitrary amount of energy was available, an infinite precision could be reached. If instead one restricts to a probe with finite energy, then a compromise between $|\alpha|, r$, and $\mu$ must be achieved. From the phase symmetry of the amplitudedamping channel, it is clear that the only relevant angular parameter is the difference between the displacement phase $\arg \alpha$ and the squeezing direction $\arg \zeta$. Hence, we can take $\zeta \equiv r \in \mathbb{R}$ and consider $\theta=\arg \alpha$ as the relevant angle. On the other hand, as also intuitively expected, it is of no use to spend energy in preparing a thermal state, that is, for any given input energy $\bar{n}$, the optimal probe state is pure. Therefore, the optimization problem can be reduced to determine two parameters, the ratio $x$ of squeezing energy to total energy and the displacement phase $\theta$. Since for a pure Gaussian state $\varrho_{0}=D\left(\alpha_{0}\right) S\left(r_{0}\right)|0\rangle\langle 0| S^{\dagger}\left(r_{0}\right) \times$ $D^{\dagger}\left(\alpha_{0}\right)$ the mean photon number is given by $\bar{n}=\sinh ^{2} r_{0}+$ $\left|\alpha_{0}\right|^{2}$ we have $\sinh ^{2} r_{0}=x \bar{n}$ and $\left|\alpha_{0}\right|^{2}=(1-x) \bar{n}$.

The evolution of the state parameters under the action of the channel may be explicitly evaluated [24]

$$
\begin{aligned}
\mu_{\phi} & =1 / \sqrt{\cos ^{4} \phi+\sin ^{4} \phi+2 \cos ^{2} \phi \sin ^{2} \phi \cosh 2 r_{0}}, \\
r_{\phi} & =\frac{1}{2} \cosh ^{-1}\left[\mu_{\phi}\left(\cos ^{2} \phi \cosh 2 r_{0}+\sin ^{2} \phi\right)\right], \\
s_{\phi} & =s_{0} \cos \phi .
\end{aligned}
$$

We can now evaluate the SLD $\Lambda(\phi)$, which corresponds 
to the optimal measurement and allows to calculate the QFI. Upon writing $\varrho_{\phi}$ in its diagonal form, $\varrho_{\phi}=$ $\sum_{k} \varrho_{k}\left|\psi_{k}\right\rangle\left\langle\psi_{k}|,| \psi_{k}\right\rangle=D(\alpha) S(r)|k\rangle$ one easily finds

$$
\Lambda(\phi)=2 \tan \phi \sum_{p q} \frac{\left\langle\psi_{q}|\mathcal{L}[a] \varrho| \psi_{p}\right\rangle}{\varrho_{p}+\varrho_{q}}\left|\psi_{q}\right\rangle\left\langle\psi_{p}\right| .
$$

A lengthy but straightforward calculation yields $\Lambda(\phi)=$ $2 \tan \phi D(\alpha) S(r) K S^{\dagger}(r) D^{\dagger}(\alpha), \quad K=\left[A a^{\dagger} a+B\left(a^{2}+\right.\right.$ $\left.\left.a^{\dagger 2}\right)-C a-C^{*} a^{\dagger}+F\right]$, where

$$
\begin{aligned}
& A=\frac{2 \mu}{1-\mu^{2}}(\mu \cosh 2 r-1), \quad B=\frac{\mu^{2}}{1+\mu^{2}} \sinh 2 r, \\
& C=\mu\left(\alpha \cosh r+\alpha^{*} \sinh r\right), \quad F=1-\frac{2 \mu \cosh ^{2} r}{1+\mu^{2}} .
\end{aligned}
$$

All the parameters in (5) are given in Eq. (3), i.e., refer to the state $\varrho_{\phi}$ after the evolution in the lossy channel. We omitted the explicit dependence on $\phi$ for brevity. Using the identity

$$
K=F^{\prime}+\tau S^{\dagger}(\eta) D(\beta) a^{\dagger} a D(\beta) S(\eta),
$$

where $F^{\prime}=F+\frac{1}{2}\left(\tau-A-2 \tau|\beta|^{2}\right), \quad \tan 2 \eta=-2 B / A$, $\tau=\sqrt{A^{2}-4 B^{2}}$, and $\beta=\left(C \cosh r+C^{*} \sinh r\right) / \mu \tau$, one sees that the eigenvectors of $\Lambda(\phi)$ are of the form $D(\alpha) S(r) S^{\dagger}(\eta) D^{\dagger}(\beta)|n\rangle$, which means that the measurement of $\Lambda(\phi)$ may be implemented with Gaussian operations and photon counting, e.g., by squeezing and displacing the state under investigation and then measuring the photon number distribution by a suitable reconstruction technique [25].

The explicit evaluation of the QFI $H(\phi)$ yields

$$
\begin{aligned}
H(\phi)= & \frac{4 z \bar{n}}{1+z(2+z+4 \bar{n} x)}\left[1-x+2 \bar{n} x+\frac{x}{z}+z\right. \\
& \left.-\frac{4 \bar{n} x^{2} z(1+\bar{n} x)}{1+z(2+z+2 \bar{n} x)}+2(1-x) \sqrt{\bar{n} x(1+\bar{n} x)}\right],
\end{aligned}
$$

where $z=e^{\gamma t}-1=\tan ^{2} \phi$, and where we have already performed the trivial optimization over the phase $\theta$, which yields $\theta=0$; i.e., the displacement should be performed along the same direction of squeezing. The optimization procedure thus reduces to maximizing the QFI $H(\phi)$ with respect to the squeezing ratio $x$. In Fig. 1 we report the renormalized QFI $H(\phi) / \bar{n}$ as a function of the squeezing ratio for different values of $\bar{n}$ and of the actual loss parameter. As is apparent from the plots in the regime of small losses and small probe energies, the optimal probe is the squeezed vacuum $\left(x_{\mathrm{opt}}=1\right)$, whereas for increasing energy there is a nonzero value of the optimal squeezing fraction $x_{\mathrm{opt}}$, which is a monotonically decreasing function of both the probe energy and the loss parameter itself. In the small energy regime, with squeezed vacuum as the optimal probe, the QFI reads

$$
H(\phi)=\frac{4 \bar{n}\left(1+z^{2}\right)}{1+2 z(1+\bar{n})+z^{2}} \simeq 4 \bar{n}+O\left(\phi^{2}\right),
$$
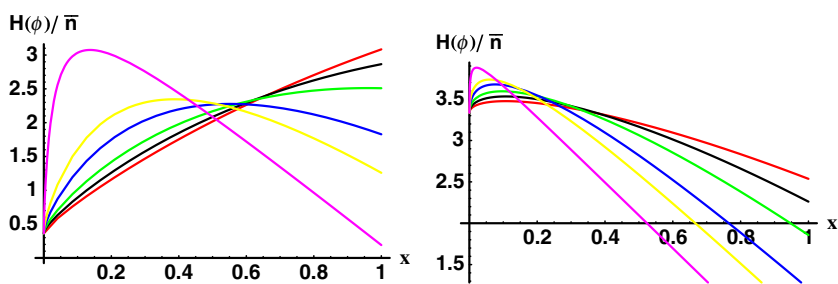

FIG. 1 (color online). Normalized quantum Fisher information $H(\phi) / \bar{n}$ as a function of the squeezing fraction of the probe state for different values of the probe energy and two values of the actual loss parameter. (Left): $\tan ^{2} \phi=0.1$. (Right): $\tan ^{2} \phi=5$. In both plots, from bottom to top in the region $x \simeq 0$, the curves for $\bar{n}=0.5, \bar{n}=1, \bar{n}=2, \bar{n}=5, \bar{n}=10$, and $\bar{n}=100$.

where the second equality expresses the attainable precision in estimating the loss parameter for weakly damping channels. In the following, we will see that this is the ultimate limit even if one has access to the environment degree of freedom. In terms of $\gamma$ the bound reads

$$
\operatorname{Var}_{\gamma}[\hat{\gamma}] \rightarrow \frac{\gamma}{\bar{n} N t}+O\left(\gamma^{2}\right) .
$$

This is a remarkable result since it is valid for any value of the loss parameter with no lower bound (see also Fig. 2). Recall, however, that squeezed vacuum probes will not be optimal for large enough values of $\gamma$.

The maximization of the QFI in the general case may be done numerically. In Fig. 2 (left) we report the log-log plot of the rescaled optimal variance $N \operatorname{Var}_{\phi}[\hat{\phi}]=1 / H_{\max }(\phi)$ as a function of the probe energy $\bar{n}$ for different values of the actual loss parameter. As is apparent from the plot the variance does not dramatically depend on the actual value of the loss parameter. The common scaling is given by $\operatorname{Var}_{\phi}[\hat{\phi}] \propto(\bar{n} N)^{-1}$.

In order to better understand the behavior in the large energy regime an asymptotic analysis is in order. Upon expanding the optimization equation $d H / d x=0$ around $z=0$ and retaining the leading order $z^{-1}$ we obtain analytically the optimal value of $x$ for small $\gamma$, which for large $\bar{n}$ can expressed as $x_{\mathrm{opt}}=(4 \bar{n} z)^{-1 / 2}+O(1 / n)$. This shows
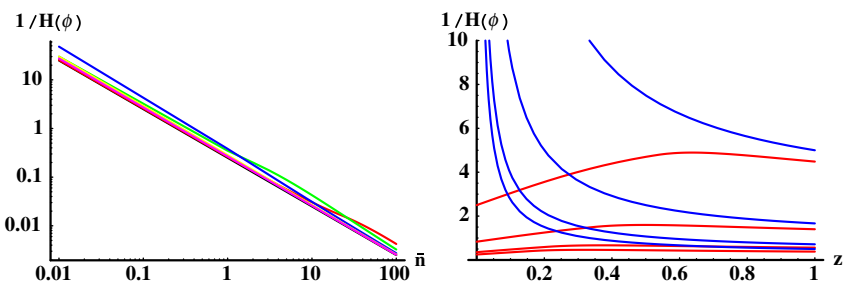

FIG. 2 (color online). (Left): Rescaled optimal variance $H^{-1}(\phi)$ as a function of the probe energy for different actual values of the loss parameter: $z=0.01,0.1,1,5,10$. The larger is $z(\phi)$ the closer is the curve to the asymptotic $H^{-1}(\phi)=(4 \bar{n})^{-1}$. (Right): Rescaled optimal variance compared to the variance obtained for coherent probe as a function of the loss parameter for different values of the probe energy: from top to bottom the curves for $\bar{n}=0.1,0.3,0.7,1$, respectively. 
that, when a large amount of energy is available, one can improve performances, besides squeezing, by employing a fraction of energy to displace the probe. The QFI in this regime reads $H(\phi)=4 z^{-1}(1-\sqrt{n z})+(2+4 n)+2 z$, which corresponds to a variance $\operatorname{Var}_{\phi}[\hat{\phi}] \rightarrow \frac{\phi^{2}}{4 N} \times$ $(1+\sqrt{n} \phi)+O\left(\phi^{4}\right)$. Finally, by rewriting this in terms of $\gamma$ we have

$$
\operatorname{Var}_{\gamma}[\hat{\gamma}] \rightarrow \frac{\gamma}{\bar{n} N t}+O\left(\sqrt{\frac{\gamma}{(\bar{n} t)^{3}}}\right)
$$

that is, the proportionality of the variance to the parameter is recovered for large $\bar{n}$. Notice that the scaling $H(\phi) \propto \bar{n}$ is valid also in the general case, as it is apparent from Fig. 2 (left).

It is worth stressing the improvement of precision with respect to that attainable with coherent states (shot-noise limit). In fact, by taking $x=0$ one easily sees that in this case the QFI reads $H(\phi)=4 \bar{n} z /(1+z)$, which corresponds to $\operatorname{Var}_{\gamma}[\hat{\gamma}] \rightarrow\left(\bar{n} N t^{2}\right)^{-1}$. In other words, the proportionality of the variance to $\gamma$ cannot be achieved using coherent probe states. In Fig. 2 (right) we report the optimal rescaled variance $H^{-1}(\phi)$ as a function of the loss parameter compared to the variance that can be obtained using coherent probes. The improvement at small values of the loss parameter is apparent. This can be intuitively understood as follows: for small values of $\gamma$ the action of the loss map $E_{\gamma}$ on a coherent probe is only that of a small displacement, whereas a squeezed vacuum is dramatically "mixed up." Hence, a squeezed state is more sensitive to small losses. Contrarily, for large values of $\gamma$ a coherent state is highly displaced whereas a squeezed vacuum state becomes close to pure again, losing its sensitivity to $\gamma$.

Let us now discuss other ways in which this performance could, in principle, be improved. The Heisenberg limit generally describes the ultimate precision attainable for parameter estimation. As we have seen above, this holds in a strict sense when one deals with unitary transformations, where the QFI characterizes the sensitivity of a state for the estimation of a parameter. However, when one deals with nonunitary transformations, this may not be true because the probe state evolves into an entangled state with the environment. The access to the degree of freedom of the environment may provide improved precision to the measurement. In the case of the loss of a channel, some of the energy present in the probe state is lost through coupling to the environment. The master Eq. (1) can be seen as the effective interaction of the mode $a$ with a second vacuum mode $b$ through a bilinear (beam-splitter-like) evolution of the form $U(\phi)=\exp \left[i \phi\left(a^{\dagger} b+a b^{\dagger}\right)\right]$. This allows a unitary representation of the process. Here we use this picture to derive the attainable precision in the hypothetic case that one had access to the bath of oscillators. In such a situation, the state under inspection would remain pure, therefore $\Lambda(\phi)=2 d \rho / d \phi$, as can be seen by taking the derivative of the identity $\rho^{2}=\rho$. The generator $G$ is given by $G=\left(a^{\dagger} b+a b^{\dagger}\right)$, and the uncertainty $\langle\Delta G\rangle^{2}$ is $\langle\Delta G\rangle^{2}=\bar{n}$. As a consequence one gets $\operatorname{Var}_{\phi}[\hat{\phi}] \rightarrow \frac{1}{4 \bar{n} N}$, which corresponds to the precision attained using squeezed vacuum probe.

In conclusion, we have shown that using Gaussian squeezed probes one can improve the estimation of the loss parameter of a bosonic noisy channel. As it holds for any single parameter quantum estimation problems, a onestep adaptive scheme is optimal to leading order, thus providing a practical way for implementation. We have shown that the optimal measurement for Gaussian probe states can be implemented by means of Gaussian operations and photon counting. Furthermore, for small losses, the estimator variance obtained by choosing appropriate probe states decreases proportionally to the loss parameter, thus providing unlimited resolution for arbitrary small losses. We have also obtained the optimal trade-off between squeezing and displacement of probe states, showing that squeezed vacuum states are optimal in the small energy limit. Finally, we have shown that even by having access to the environment, one cannot improve the performance of our scheme.

This work has been supported by MIUR Project No. PRIN2005024254-002, MCyT Project No. FIS200501369, and Consolider-Ingenio 2010, project "QOIT".

*Also with ISI Foundation, Torino, Italy.

[1] J. M. Geremia et al., Phys. Rev. Lett. 91, 250801 (2003).

[2] H. Cramer, Mathematical Methods of Statistics (Princeton University, Princeton, NJ, 1946).

[3] C.W. Helstrom, Quantum Detection and Estimation Theory (Academic, New York, 1976).

[4] A.S. Holevo, Probabilistic and Statistical Aspects of Quantum Theory (North-Holland, Amsterdam, 1982).

[5] S. Braunstein et al., Phys. Rev. Lett. 72, 3439 (1994).

[6] S. Braunstein et al., Ann. Phys. (N.Y.) 247, 135 (1996).

[7] L. Maccone, Phys. Rev. A 73, 042307 (2006).

[8] A. S. Holevo, Rep. Math. Phys. 16, 385 (1979).

[9] M. D’Ariano et al., Phys. Lett. A 248, 103 (1998).

[10] A. Monras, Phys. Rev. A 73, 033821 (2006).

[11] C. W. Helstrom, Found. Phys. 4, 453 (1974).

[12] G. J. Milburn et al., Phys. Rev. A 50, 801 (1994).

[13] G. Chiribella et al., Phys. Rev. A 73, 062103 (2006).

[14] M. D'Ariano et al., J. Opt. B 3, 337 (2001).

[15] M. Hotta et al., Phys. Rev. A 72, 052334 (2005); J. Phys. A 3914465 (2006).

[16] A. Fujiwara, Phys. Rev. A 63, 042304 (2001).

[17] J. Zhenfeng et al., quant-ph/0610060.

[18] V. D’Auria et al., J. Phys. B 39, 1187 (2006).

[19] P. Grangier et al., Phys. Rev. Lett. 59, 2153 (1987).

[20] E. S. Polzik et al., Phys. Rev. Lett. 68, 3020 (1992).

[21] G. M. D’Ariano et al., Phys. Rev. Lett. 87, 270404 (2001).

[22] A. Acín, Phys. Rev. Lett. 87, 177901 (2001).

[23] R. Gill and S. Massar, Phys. Rev. A 61, 042312 (2000).

[24] A. Serafini et al., J. Opt. B 7, R19 (2005).

[25] G. Zambra et al., Phys. Rev. Lett. 95, 063602 (2005). 